\title{
Genetic Programming for Modeling a Nonlinear Optical System
}

\author{
Amr Radi" and S. K. Hindawi** \\ Physics Department, Faculty of Science, Ain Shams University \\ *Amr_Radi@hotmail.com ***skhindawi@hotmail.com
}

Genetic Programming (GP) is used to construct a model that describes the dependency of the refractive index of a $\gamma$-irradiated optical GRIN fiber on both the wavelength and the dose of the $\gamma$-radiation. The GP based discovered function shows good agreement with the previously published experimental data carried at a certain wavelength for different $\gamma$ radiation doses.

\section{Introduction:}

Most systems in the real world are essentially nonlinear and time-dependent [1]. Genetic Programming (GP), nowadays, proves a powerful technique for easy handling mathematical models, as there is no special knowledge necessary to search a solution, moreover it can cope with the changes of various conditions. GP is usually used to automatically build a model, e.g. a physical system, from its input and output data without any knowledge about the system itself.

In a previous work, one of the authors [2] handled the same problem with Neural Networks (NN). The $\mathrm{NN}$ is a method for global modeling, but, unfortunately, it cannot give simple and elegant model representation. Moreover, $\mathrm{NN}$ is less powerful in revealing the system dynamic laws and is difficult to be integrated with the pre-discovered knowledge on nonlinear systems [3].

On the other hand, GP modeling became one of the researcher's interests in modeling of high energy physics [4], and in Automated Re-Invention of Six Patented Optical Lens Systems [5]. On the top of this, GP have succeeded in the field of automatically defining functions [6]. Also, Oakley [7] used GP to evolve equation fitting of the chaotic time series produced by Mackey-Glass equations, and Iba et al [8] used GP for system identifications. 
The effect of Gamma radiation has been previously studied [9], on the optical parameters of the GRIN optical fiber, with different doses in the range 0.1 - 1.0 MGy. In that work, a modified Two-Pole Sellmeier Dispersion relation is adopted, fitted to the experimental data of the refractive index, and used to deduce a dose-dependent formula.

Using the capability of the GP, refractive index can be modeled as a function of both the wavelength and the radiation dose. For this purpose, GP is fed with wavelengths and radiation doses while the output is the refractive index.

The following sections provide a brief introduction to Two-Pole Sellmeier Dispersion formula, description of the selected GP structure, and finally results are discussed.

\section{Two-Pole SELLMEIER Dispersion Formula:}

An accurate dispersion formula (find the refractive index $n$ ) that can be applied over a wide range of wavelengths is Two-Pole Sellmeier Dispersion formula in the form [10]

$$
n^{2}(\lambda)=A+B \lambda^{2} /\left(\lambda^{2}-C\right)+D \lambda^{2} /\left(\lambda^{2}-E\right)
$$

$\lambda$ is the wavelength measured in micrometers and $A, B, C, D$, and $E$ are called the dispersion parameters of the fiber materials with $\mathrm{E}=100 \mathrm{um}^{2}$. Values of those parameters [9], at different radiation doses, are given in Table 1, at $25^{\circ} \mathrm{C}$.

Present proposal is to use GP for finding a function that represents the refractive index $\mathrm{n}^{2}(\lambda, \gamma)$ depending on wavelengths and radiation dose. It is expected that this function will prove better matching with experimental data.

Table 1: Sellmeier Dispersion Parameters at Different Doses (MGy) [9]

\begin{tabular}{|l|l|l|l|l|}
\hline Dose & A & B & C & D \\
\hline 0.0 & 1.329670 & 0.829166 & 0.0110495 & 0.985996 \\
\hline 0.1 & 1.330103 & 0.829484 & 0.0110555 & 0.986084 \\
\hline 0.3026 & 1.330046 & 0.830828 & 0.0110611 & 0.986430 \\
\hline 0.462 & 1.331487 & 0.830612 & 0.0110605 & 0.986410 \\
\hline 0.805 & 1.332633 & 0.833233 & 0.0110883 & 0.987561 \\
\hline 1.0 & 1.336000 & 0.836167 & 0.0111100 & 0.987881 \\
\hline
\end{tabular}




\section{Modeling by GP:}

\subsection{Model Building}

Building a prediction model using GP has many advantages: to handle mathematical model is easy, special knowledge is unnecessary to search a solution, and it can cope with the change of various conditions.

GP handles tree-structured chromosome that represents the mathematical model of the system. As shown in Fig. (1), nodes involved in the tree-structured chromosome belong to two categories: one involves input data (leaves A and B), the other involves operators $\{+, *\}$ (inner nodes of the tree).

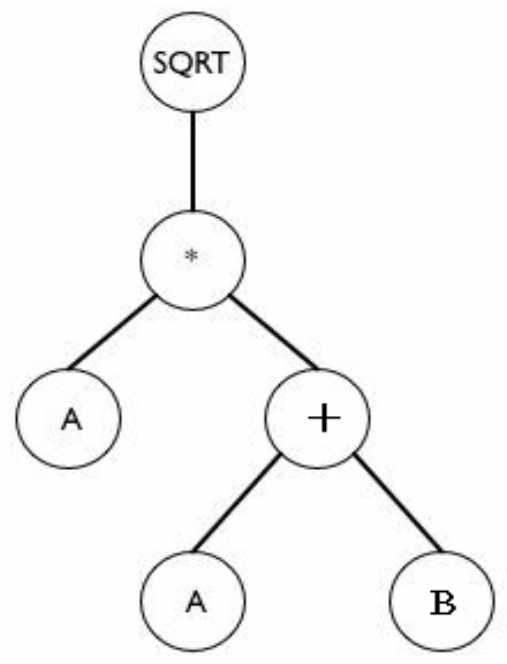

Fig. (1): Tree representation of the equation square root * $(A,+(A, B))$ i.e. $(A *(A+B)$

\subsection{Procedure}

GP $[6,11,12]$ evolves a population of computer programs, which are possible solution to a given optimization problem, using the Darwinian principle of survival of the fittest. It uses biologically inspired operations like reproduction, crossover and mutation. Each program or individual on the population is generally represented as a tree composed of functions (operators) and data terminals (leaves) appropriate to the problem domain. The input set of functions and terminals have to satisfy the closure and sufficiency prosperities. The sufficiency closure property requires that the set of functions in and the set of terminals be able to express a solution of problem The function set may include standard arithmetic operators, logical operators, mathematical functions and specific functions. The terminal set usually consists of feature variables and constants. Each individual in the population is assigned a fitness value, which 
quantifies how well it performs in the problem environment. The fitness value is computed by a problem dependent fitness function.

A typical implementation of GP involves the following steps:

Step: 1 GP begins with a randomly generated population of solutions.

Step: 2 A fitness value is assigned to each solution of the populations.

Step: 3 A genetic operator is selected probabilistically as follows:

Case i) If it is the reproduction operator; then an individual is selected (we use fitness proportion-based selection) from the current population and it is copied into the new population. Reproduction replicates the principle of natural selection and survival of the fittest.

Case ii) If it is the crossover operator; two individuals are selected. We use tournament selection where number of individuals is taken randomly from the current population, and out of these, the best two individuals (in terms of fitness values) are chosen for the crossover operation. Then, we randomly select a sub-tree from each of the selected individuals and interchange these two sub-trees. These two offspring are included in the new population. Crossover plays an essential role in the evolutionary process.

Case iii) If the selected operator is mutation; then a solution is (randomly) selected. Now, a sub-tree of the selected individual is randomly selected and replaced by a new randomly generated sub-tree. This mutated solution is allowed to survive in the new population. Mutation maintains diversity.

Step: 4 Continue step: 3, when the new population renders solutions, this completes one generation.

Step: 5 If GP will not converge; steps: $2-4$ are repeated till a desired solution (may be $100 \%$ correct solution) is achieved. Otherwise, the GP operation is terminated after a predefined number of generations.

\section{Proposed GP}

The authors approach is to be adopted the experimental data [9] (the wavelengths $\lambda$ and radiation dose values $\gamma$ ) to produce the refractive index for each case. Those are used as input variables to find the suitable function that can describe the experimental data relations.

The fitness function is calculated as a negative value of the total absolute performance error of the discovered function on the experimental data set, i.e. a lower error must correspond to a higher fitness. The total performance error can be defined over all the experimental data $(\mathrm{j}=1 \ldots, \mathrm{n})$ set as:

$$
E=\Sigma_{j}\left|X_{j}-Y_{j}\right|^{2}
$$


Where $\mathrm{n}$ is the data size, $\mathrm{X}_{\mathrm{j}}$ represents the experimental data and $\mathrm{Y}_{\mathrm{j}}$ represents the calculated data. The running process stops when the error is reduced to an acceptable level $\mathrm{E}=0.00001$.

\section{Results}

Our representing GP was run for 500 generations with a maximum population size of 1000 . The operators (and selection probability) were: crossover probability as 0.9 and mutation probability as 0.01 . The function set was $\{+,-, *, \backslash, \log , \log 2, \cos , \sin \}$, and the terminal set was \{random constancy from 0 to $10, \lambda, \gamma\}$. The "full" initialization method was used with an initial maximum depth of 27 , and tournament selection with size 6 .
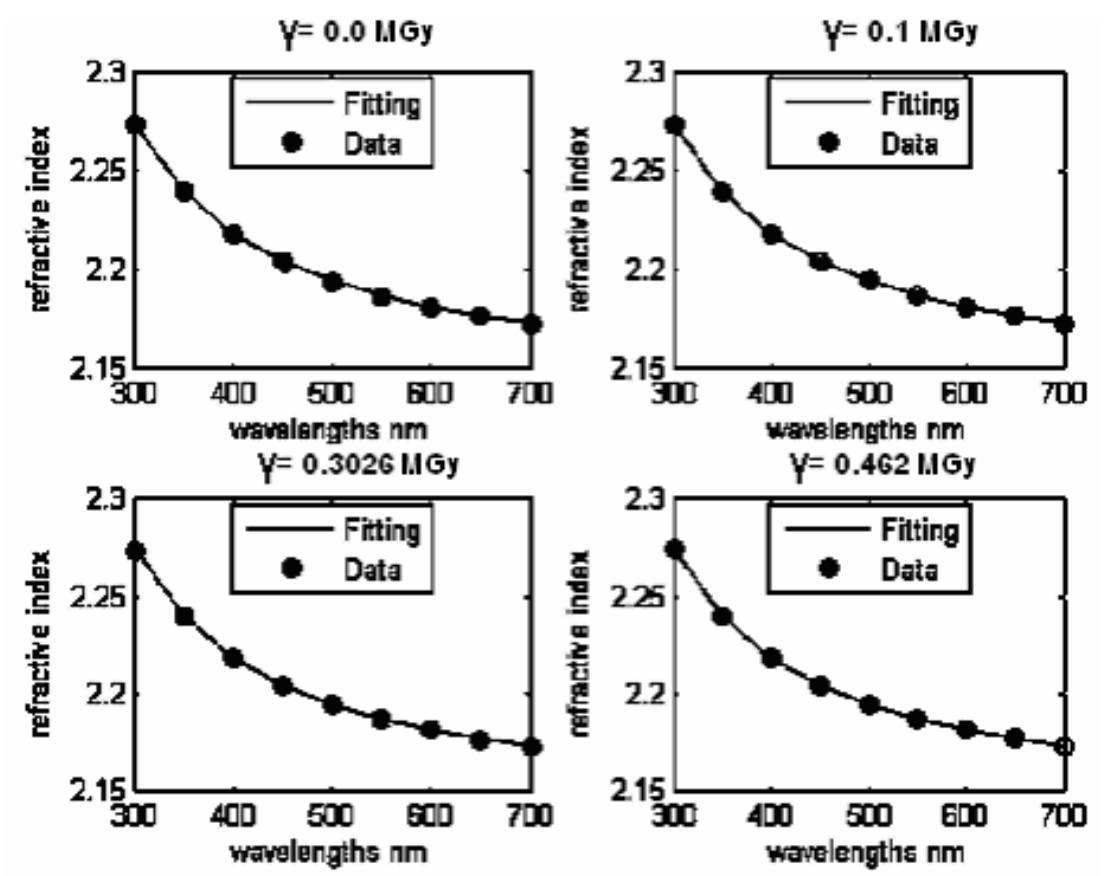

Fig. (2): Experimental and Trained Refractive Index

The input data for training are the wavelengths $\lambda=300-700 \mathrm{~nm}$ with $50 \mathrm{~nm}$ step, and radiation dose values $\gamma=0.0,0.1,0.3026$ and $0.462 \mathrm{MGy}$. Each refractive index $\mathrm{n}$ corresponds to a certain wavelengths $\lambda$ at different radiation doses $\gamma$ are shown in Fig. (2). The discovered function (see Appendix) has been trained to associate the input patterns to the target output patterns for the above wavelengths. 
After running the GP, the discovered function has been used to predict refractive index, corresponding to dose value $\gamma=0.802$ and $1.0 \mathrm{MGy}$ with the same $\lambda=300-700 \mathrm{~nm}$. The experimental and predicts refractive indexes are illustrated in Fig. (3).
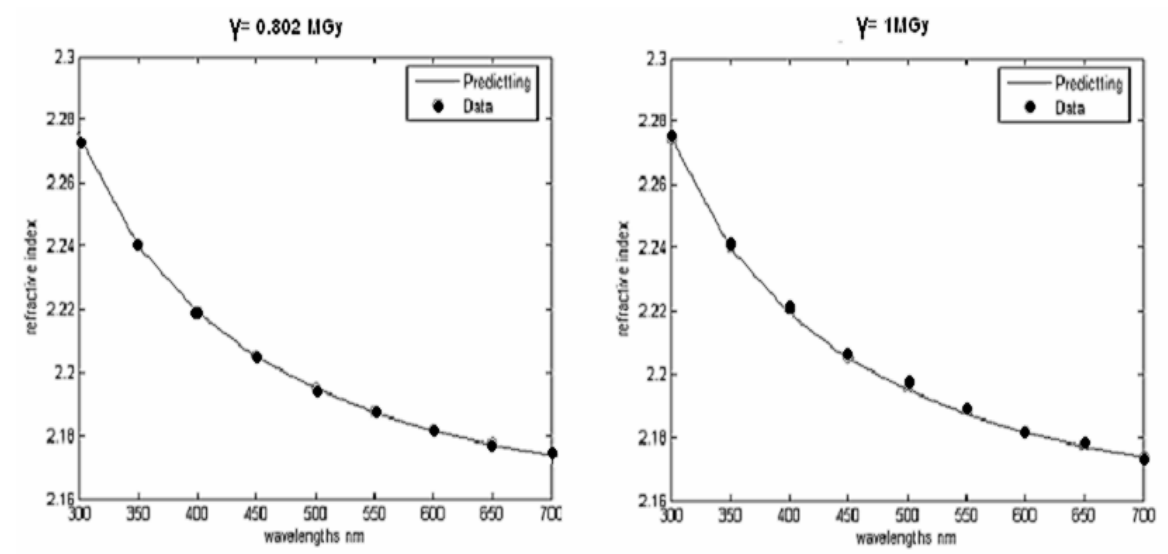

Fig. (3): Experimental and Predicted Refractive Index

\section{Conclusions:}

Genetic programming has been used to model the Sellmeier Dispersion formula to depend on the wavelength and the radiation dose values. The discovered function shows an excellent agreement with the published experimental data [9]. Moreover, it is capable of predicting experimental data for Dispersion formula that are not used in the training session. Finally, this leads to conclude that GP can be applied effectively in the field of optics.

\section{Appendix :}

The best atomic expression for representing the input data is:

$f(\lambda, \gamma)=$

$*(+(\log (\sin (\log ((/(\log 2(+(/(/(\log (\log (\lambda)), 10), \log (0.72349)), \lambda)), \log 2(/(\log 2(\lambda),(/($ $\log 2(+(/(/(\log (\log ((\log 2(\lambda), 10))), 10), \log (0.72349)), \lambda)), \log 2(/(\log 2(\lambda), 10))), 10))$

)$), 10)))),(\cos (\cos (\sin (/(\log 2(+/ /(/(\log (\log (\lambda)), 10), \log (\sin (\log ((/(\log 2(+/ /(/ / \log (\operatorname{lo}$ $\mathrm{g}(\lambda)), 10), \log (0.72349)), \lambda)), \log 2(/(\log 2(\lambda),(/(\log 2(+(/ / /(\log (\log ((\log 2(\lambda), 10))), 1$ $0), \log (0.72349)), \lambda)), \log 2(((\log 2(\lambda), 10))), 10)))), 10))))), \lambda)), 10)))), \log (*(\log 2(0.8$ $5892), \sin (0.72349))))), /\left(\gamma,{ }^{*}(*(*(\lambda, 1.342)(10, \gamma)))\right.$ 
Where $\lambda$ and $\gamma$ are the wavelengths and the radiation dose values, respectively, the operator $(\mathrm{O})$ is defined as $\mathrm{O}(\mathrm{A}, \mathrm{B}) \equiv \mathrm{A} \mathrm{O} \mathrm{B}$; for the used operators $\{+,-, *, 1$, $\log , \log 2, \cos , \sin \}$

\section{References}

1. K. J. Astrom and P. Eykhoff; "System Identification: A Survey", Automatica vol.7, (1971).

2. S. K. Hindawi; "NN Modeling the Gamma Irradiated Optical Fiber", Egyptian Computer Sci. J., Vol. 28, No. 3, (2006).

3. J. R. Koza, M. A. Keane, M. J. Streeter, Mydlowec William, Yu Jessen, and Lanza Guido; Genetic Programming IV: Routine HumanCompetitive Machine Intelligence. Kluwer Academic Publisher, (2003).

4. J. M. Link, et. al. (FOCUS Coll.); "Application of Genetic Programming to High Energy Physics Event Selection", Nucl. Instrum. Meth. A 551, 504, (2005).

5. J. R. Koza, S. H. Al-Sakra, Lee W. Jone; "Automated Re-Invention of Six Patented Optical Lens Systems using Genetic Programming", Genetic and Evolutionary Computation Conference (GECCO)'05, June 25-29, Washington, DC, USA, 20, (2005).

6. J. R. Koza; "Genetic Programming: Programming of Computers by Means of Natural Selection", Cambridge, MA: MIT Press, (1992).

7. E. H. N. Oakley; Two scientific applications of genetic programming: stack filters and non-linear equation fitting to chaotic data. In K. E. Kinnear Jr. (editor). "Advances in Genetic Programming". Cambridge, MA: The MIT Press. Pages 369 - 389, (1994).

8. H. Iba, H. deGaris, and T. Sato; "Recombination Guidance for Numerical Genetic Programming", in Proc. of $2^{\text {nd }}$ International Conference on Evolutionary Computation, IEEE Press, (1995).

9. M. Medhat, S. El-zaiat, Saleh M. Abdou, A. Radi and M. Omar; "Interferometric Determination of Gamma Radiation Effects on Optical Parameters of a GRIN Optical Fibre", J. Opt. A: Pure Applied Opt. 4485 490, (2002).

10. G C Bhar; Applied Optics, 15, 305, (1976).

11. W. Banzhaf, P. Nordin, R. E. Keller and F.D. Francone; "Genetic Programming: An Introduction on the Automatic Evolution of Computer Programs and Its Applications", Morgan Kaufmann Publishers, Inc., San Mateo, CA: Morgan Kaufmann, (1998).

12. I. Yoshihara, S. Sato; "Nonlinear Model Building Method with GA and $G M D H^{\prime}$, IPSJ, AI-105, pp.1-6, (1996) (in Japanese). Advances in Genetic Programming, (ed. Kenneth E. Kinnear, Jr.), MIT Press, (1994). 